\title{
The caecocolonic junction in humans has a sphincteric anatomy and function
}

\author{
M S Faussone Pellegrini, L Ibba Manneschi, L Manneschi
}

\begin{abstract}
Sphincteric anatomy and function are present at the caecocolonic junction in several mammals. In humans, radiologists and endoscopists have respectively reported a circumferential contraction and a prominent ileocaecal fold at the border area between the caecum and the ascending colon. Anatomical findings on necropsy material failed to confirm its presence. Microscopic studies on surgical specimens showed the existence of muscular and innervational patterns different from those of adjacent areas. The aim of this work was to confirm the existence of a specialised fold at the caecocolonic junction in humans and to ascertain its role by carrying out a study of functional anatomy. Pancolonoscopies were performed on 100 patients and ileocaecal fold behaviour was observed before and after mechanical stimulation. Isolated ileocaecocolonic regions, surgically obtained, were filled with a fixative solution to study their macro and microscopic morphology after stimulation. Endoscopically, the ileocaecal fold was semilunar or circular in shape and spontaneous or evoked spasms occurred in 52 patients. A prominent circular fold could be seen in surgical specimens after stimulation. The entire muscle coat deeply penetrated this fold, showing the features characteristic of the ileocaecal junction. In particular, the inner portion of the circular muscle showed a peculiar arrangement and was thicker than elsewhere. These results show that in humans the caecocolonic junction is provided with a sphincter morphology and function. Little is known about its physiological relevance in ileal flow accommodation and caecal filling and emptying but it should not be underestimated with regard to some colonic motility disorders.

(Gut 1995; 37: 493-498)
\end{abstract}

Keywords: anatomy, caecocolonic junction, colonoscopy, endoscopy, sphincter.

In most mammals the terminal ileum joins the large intestine end to side, marking the beginning of the ascending colon in the distal direction and of the caecum in the proximal direction. For this reason, this border area can be called caecocolonic junction (CCJ).

Studies on the CCJ anatomy and its function in several animal species showed differences in caecum size and CCJ specialisation, depending upon the type of their diet and digestion. For instance, in herbivorous animals the caecum is enormous and separated from the colon by a well developed caecocolonic sphincter. In carnivorous animals, such as the dog, notwithstanding that the caecum is less developed, a caecocolonic sphincter is also present. ${ }^{1}$ In omnivorous animals, such as humans, the caecum is extremely short and, at the CCJ, a sphincteric organisation seems lacking. Consequently, until now, the morphology and function of the CCJ has been scarcely considered in humans.

Radiologists, however, have reported a sphincter located immediately above the ileocaecal junction (ICJ), ${ }^{2}$ at the CCJ level, and separating the caecum from the ascending colon. On radiological examination, this area corresponds to the first encountered circumferential fold and has been called Busi's sphincter. ${ }^{2}$ Similar to other colonic circumferential or functional folds, it is seen as a circular contraction but it always occurs in a fixed place of the colonic wall. ${ }^{34}$

More recently, endoscopic studies have added new information in favour of a possible sphincteric part played by the CCJ in humans. In fact, it has been reported that a fold, the so called ileocaecal fold, approximately located at the CCJ, 5-8 cm from the caecal pole, has a pronounced tendency to spasmodic activity. ${ }^{5}$ This fold is not always the lowest one, but is usually the most prominent ${ }^{6}$ and shows a circular $^{5}$ or, less commonly, a semilunar shape. ${ }^{7}$

Anatomical findings, mainly based on necropsy material, did not confirm the presence of any specialised area at the CCJ, except for the ICJ lips and frenula. ${ }^{8-12}$ On the contrary, microscopic findings of surgical specimens of the CCJ, that is the caecocolonic wall around and opposite to the ICJ opening, showed a peculiar arrangement of the circular muscle layer. ${ }^{13}$ Furthermore, VIPergic and nitrergic innervational patterns are different from the neighbouring areas and similar to those of the ICJ. ${ }^{14} 15$

On the basis of these functional and microscopic findings, a specialised area, with a sphincteric role, located at the CCJ level, seems to be present in humans.

The aim of our work was to confirm this role both anatomically and functionally. Therefore, we performed a study of functional anatomy by using two different procedures to evaluate the CCJ stimulation: (a) endoscopic examination of the caecocolonic region in vivo, before and after mechanical stimulation of the ileocaecal fold; (b) macro and microscopic examination of the ileocaecocolonic region from surgical 


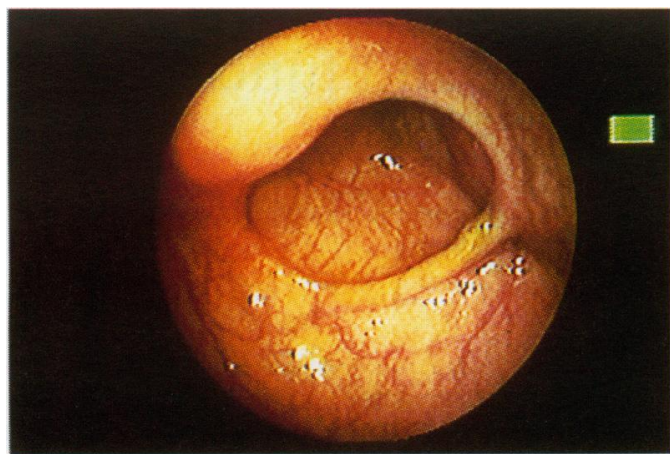

Figure $1 \mathrm{~A}$

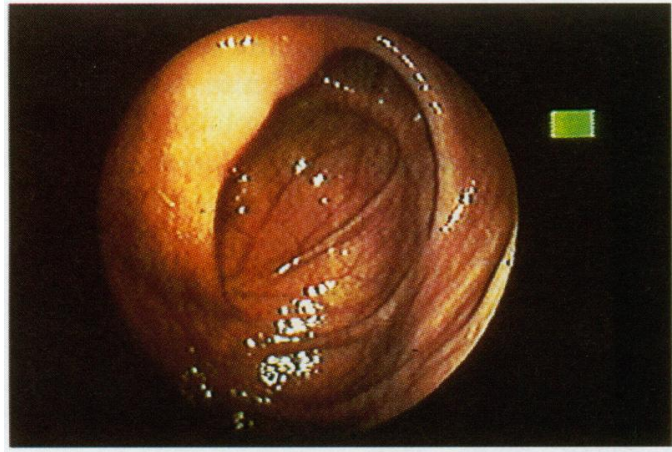

Figure $1 B$

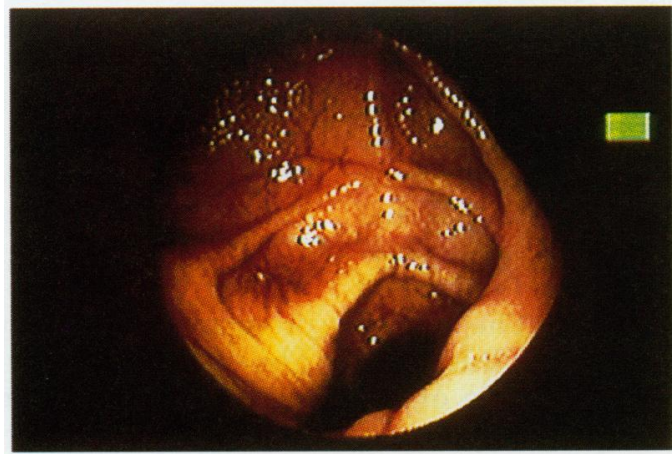

Figure $1 C$

Figure 1: Endoscopy. (A) Meocaecal fold with a circular shape; (B) ileocaecal fold with a semilunar shape; (C) two ileocaecal folds, originating and diverging from the ICF lips, can be seen.

specimens after closing the ascending colon and injecting a fixative solution through the terminal ileum. The injection of this solution had two aims: (a) to stimulate, by filling the terminal ileum, the onset of the motor activities regulating in vivo the intestinal content transit through the ICJ, and (b) to maintain, by using a fixative solution, the morphology of this region after the stimulation.

\section{Methods}

\section{Endoscopy}

A consecutive series of 100 pancolonoscopies were performed without pharmacological treatment (sedation, analgesia, antispasmodics) in patients who gave their informed consent (59 males and 41 females; age range: 23-70 years; mean age: 56$)$. No organic disorder in the right

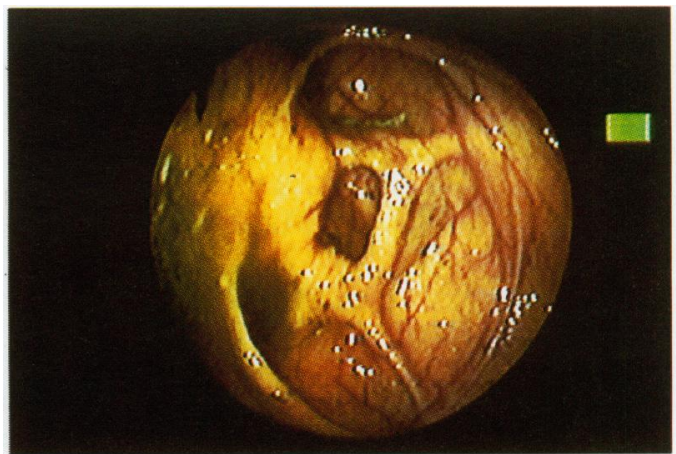

Figure $2 A$

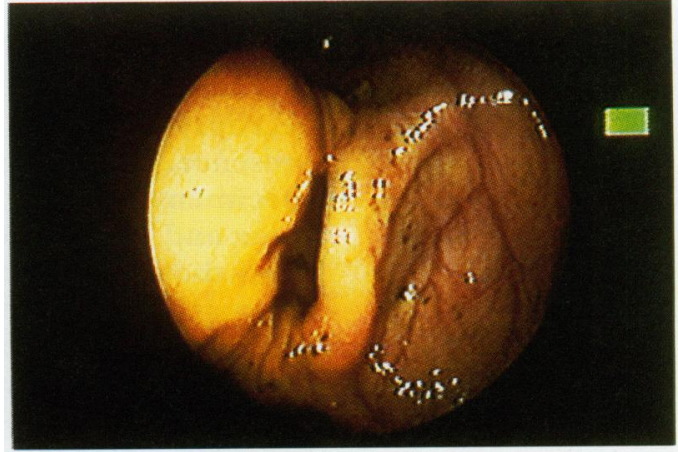

Figure 2B

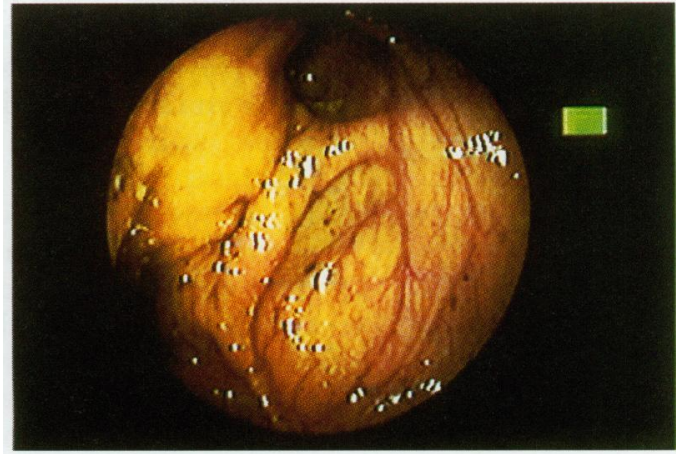

Figure 2C

Figure 2: Endoscopy. Stimulated ileocaecal fold. (A) $A$ spasm is occurring; (B) an occlusive spasm has occurred; (C) the colonic lumen is completely closed.

colon or ileum and no clinical evidence of irritable colon syndrome were present.

The caecum and the ileocaecal area were scanned and then observed for two minutes without pushing or pulling the endoscope, and without inflating or aspirating the colon. During observation, a spontaneous spasm of the ileocaecal fold was seen in five cases. In the other 95 patients a spasm was evoked by mechanical stimulation: the ileocaecal fold and the intestinal wall around and on the opposite side of the fold were pushed two or three times with biopsy forceps. After stimulation, a maximum of three minutes was allowed to elapse to see whether or not a spasm occurred. The resolution time of both spontaneous and evoked spasms was then recorded.

\section{Anatomy}

Surgical specimens were obtained from six 


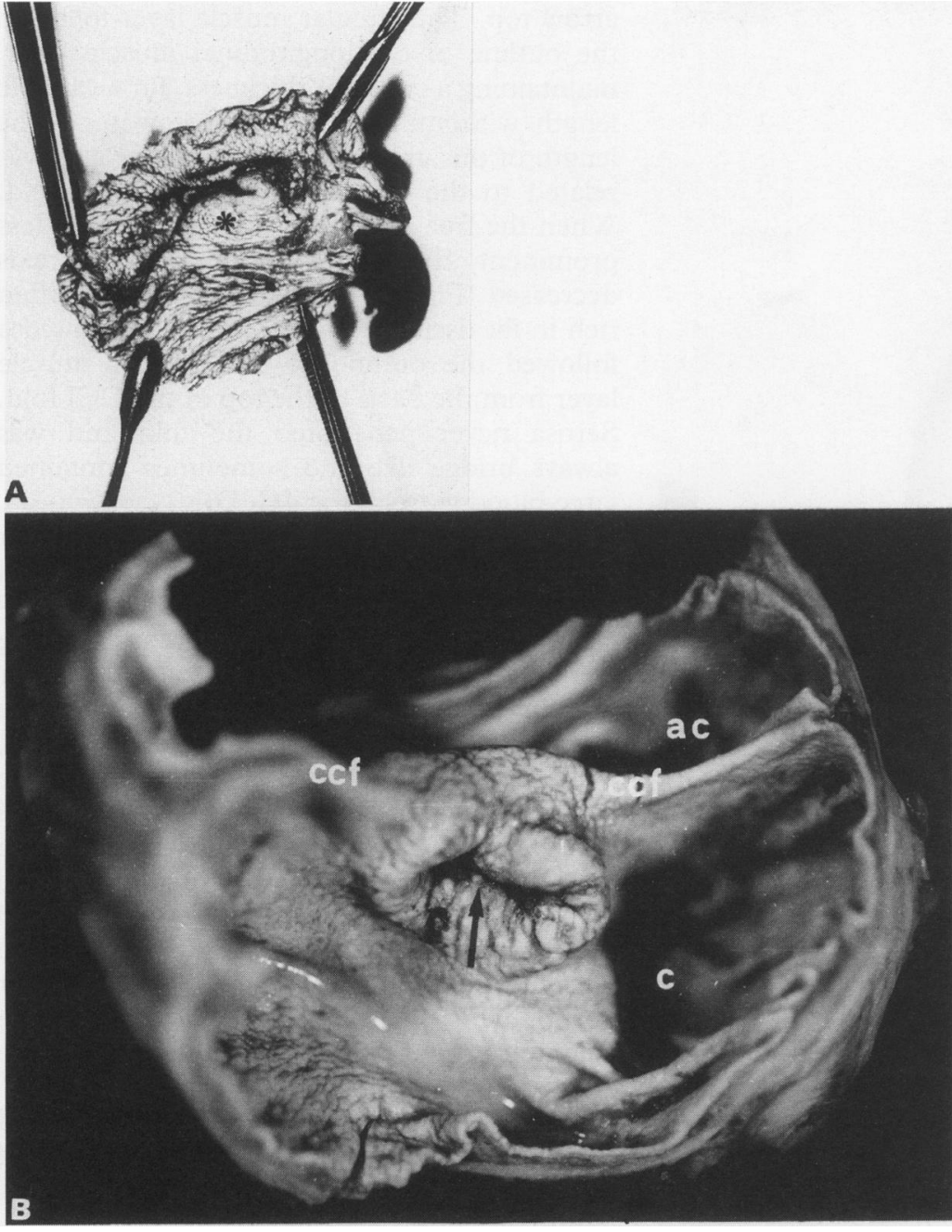

Figure 3: Isolated ileocaecocolonic region. (A) Ascending colon cut along a transverse plane. A prominent and thick circular fold (asterisk) occludes the colonic lumen; (B) cut has been performed along the anterior taenia. ccf: Caecocolonic fold, c: caecum, ac: ascending colon. The arrow points to the ICf opening towards the caecal pole.

patients (three males and three females; age range 57-68 years; mean age: $60 \cdot 16)$ undergoing right hemicolectomy for Dukes's A carcinoma of the proximal transverse colon. All patients gave their informed consent.

Immediately after surgery, the last $10 \mathrm{~cm}$ of the terminal ileum, the entire caecum, and 10 $\mathrm{cm}$ of the ascending colon were isolated from the remaining colon. All these segments had a normal appearance and were histologically free from tumour or inflammation. The proximal and distal ends of every segment were closed by clamps before the filling stimulation. These segments were then filled up by injecting a Bouin's fixative solution through the terminal ileum and immersed in the same solution for 12 hours.

For macroscopic examination, every segment was opened by cutting the wall along different planes. Three consecutive cuts were performed. The first one was made along a transverse plane at the ascending colon, $5 \mathrm{~cm}$ from the ICJ upper border (the ileocolonic lip), to have an overview of the CCJ similar to that of the endoscopist. The second cut was performed along the anterior taenia starting from the caecum towards the ascending colon to identify the topographical relation between the CCJ and the ICJ. The third cut was made along the mesenterial border of the colon and the caecum and along the mesenterial and antimesenterial borders of the ileum, to evaluate the spatial relation between the ICJ lips and the CCJ, and the extension of the second with regard to the longitudinal axis of the colon.

The circumferential area corresponding to the CCJ identified in this way could be thus isolated. Specimens, $2-3 \mathrm{~cm}$ long and $1 \mathrm{~cm}$ wide, were obtained by cutting this area along the colonic longitudinal axis and were histologically examined. Taenia were cut in some specimens, whereas serosa was cut both in taenial and extrataenial segments. Specimens were dehydrated in ethanol and embedded in paraffin wax. Longitudinal sections, 5-7 $\mu \mathrm{m}$ thick, were stained with either haematoxylin and eosin or PAS reaction and observed under a light microscope. Photographs of the entire sections were also taken.

\section{Results}

\section{Endoscopy}

In all patients $(100 \%)$ the ileocaecal fold was easily identifiable. Proceeding from the caecal pole, this fold was the first one encountered in 61 cases, while it was preceded by one or two smaller folds in 39 cases. This ileocaecal fold had a circular shape in 75 patients (Fig 1A) and semilunar in the remaining 25 (Fig 1B). Sometimes, two or three folds, could be seen originating and then diverging from ICJ. The most prominent fold always started from the ileocolonic lip of the ICJ (Fig 1C). When the spasm occurred, the ICJ moved towards the caecal pole. A complete spasm of the ileocaecal fold occurred in 52 patients, all of whom had a circular shaped fold (Fig 2A-C). The spasm was spontaneous in five and mechanically evoked in the other 47. The spasms lasted from one minute and 50 seconds to four minutes and 30 seconds, with an average time of two minutes and 50 seconds. No spontaneous or evoked spasm could be seen in any of the patients with a semilunar fold and in 23 patients with a circular fold.

\section{Anatomy}

Macroscopic findings - during the filling of the isolated ileocaecocolonic region, an anular stricture could be seen all around the colonic wall, immediately distal to the ICJ, marking the beginning of the ascending colon. In three cases, after the removal of the colonic clamp and a transverse cut of the ascending colon, a prominent and thick circular fold could be seen at the CCJ level (Fig 3A). This fold had a sphincteric appearance and occluded the lumen almost completely. In the other three cases, a thick fold with a semilunar shape and a smooth luminal contour only partially reduced the CCJ lumen. When the colon had been cut along the anterior taenia, these folds were seen starting from the ileocolonic lip of ICJ. Thus, the CCJ fold clearly defined the border area between the caecum and the ascending colon (Fig 3B). Frenula, usually described at this 


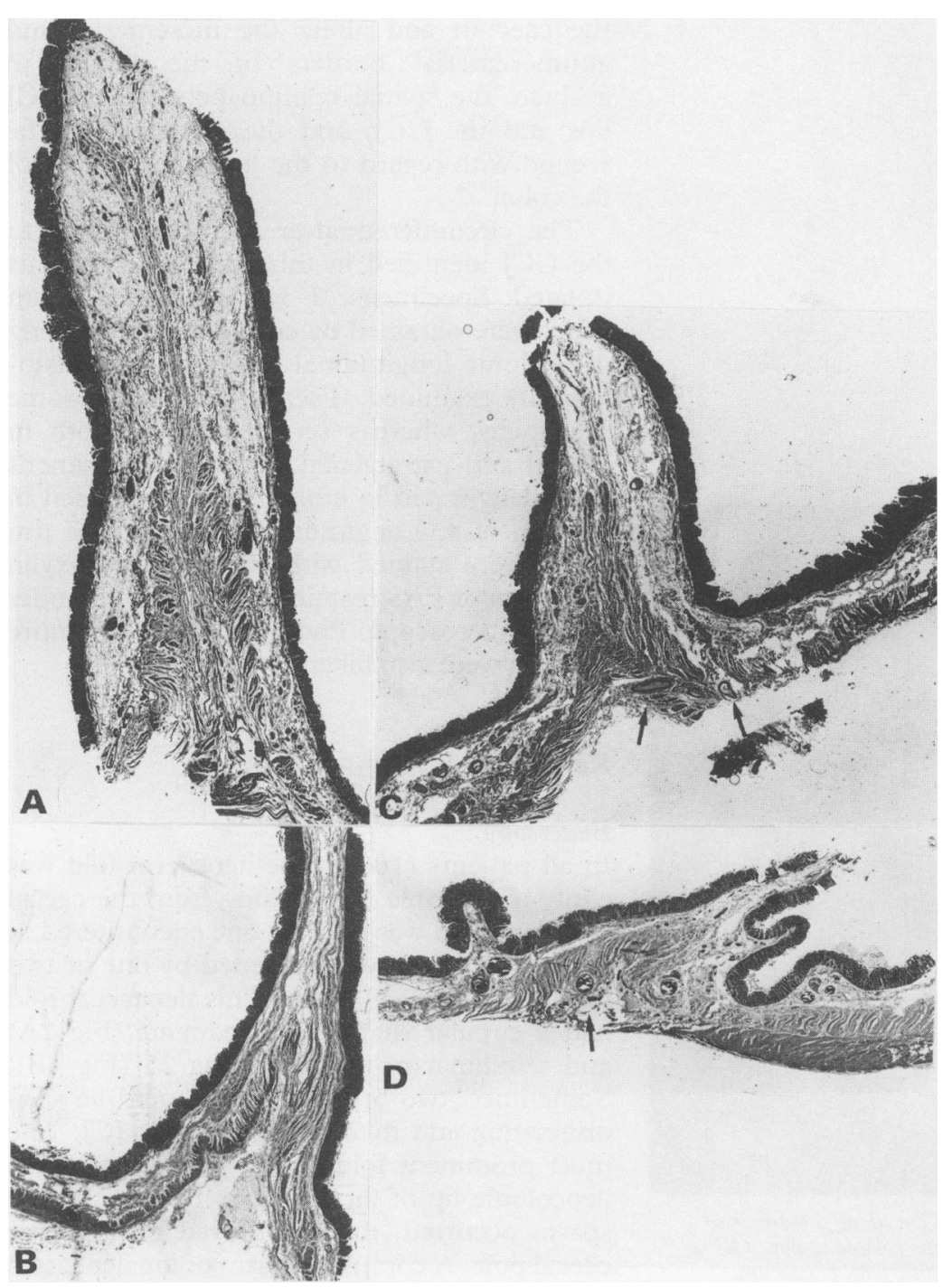

Figure 4: Longitudinal sections of the CCF wall showing the CCF structure. (A) Near the IC7. Haematoxylin and eosin, original magnification $\times 7 ;(B)$ and $(C)$ distant from the ICF. PAS, original magnification $\times 7$; (D) opposite to the ICF. Haematoxylin and eosin, original magnification $\times 7$. Arrows point to large blood vessels at the serosa level.

level in necropsy material as short and thin structures anchoring ICJ lips to the colonic wall, could not be identified. The ICJ had an oval or circular shape and always showed an open orifice (Fig 3B). In one case, two folds could be distinguished at the CCJ level, after cutting the colon and the ileum along the mesenterial and antimesenterial borders. Both folds had a common origin from the ICJ. The distal fold was prominent and had a semilunar shape, while the proximal one, in contrast, was smaller and shorter.

Microscopic findings - all specimens obtained from the CCJ, including the CCJ fold and the adjacent wall, showed a different muscle architecture from that of all other parts of the colon. In fact, the examination of the colonic wall, longitudinally sectioned (Fig 4A-D), showed that the whole muscle coat deeply penetrated the CCJ fold, assuming the shape of an arrowhead with the basis towards the serosa and the top towards the free margin of the fold. The longitudinal muscle layer, followed by the myenteric plexus, formed the inner part of the arrow. It had a triangular shape, with a large basis corresponding to the arrow base and an extremely thin apex proceeding towards the arrow top. The circular muscle layer followed the outline of the longitudinal muscle layer, maintaining a constant thickness, for a variable length without reaching the arrow top. The length of this arrow shaped muscular axis was related to the prominence of the CCJ fold. When the free margin of the fold became less prominent, the length of the muscular axis decreased (Fig 4A-D). The submucosa, often rich in fat tissue (Fig 4A, C), and the mucosa followed the outline of the circular muscle layer from the basis to the top of the CCJ fold. Serosa never penetrated the fold and was always bridge like and sometimes contained large blood vessels (Fig 4C, D).

An inner and an outer portion of the circular muscle layer could be easily identified within the CCJ fold (Fig 4A-D) and at a short distance from it, both distally and proximally (Fig 5). This peculiar arrangement of the circular muscle layer was identical to that of the unstimulated surgically obtained CCJ. ${ }^{9}$ In fact, wide septa of connective tissue separated the two circular muscle layers and split up the inner one into anastomosing muscle cords (Fig $6 \mathrm{~A})$. The outer portion was roughly three to four times thicker than the inner portion and the smooth muscle cells of the inner portion were more PAS positive than those of the outer portion (Fig 6B). Both the caecal and the colonic portions of ICJ lips showed the same architecture of the CCJ fold (Fig 7).

These features were identical in taenial and extrataenial specimens and did not change if serosa or taenia had been previously cut (compare Fig 4B with Fig 4D).

\section{Discussion}

Endoscopic findings confirmed the presence of folds differently shaped in each colonic segment. The folds seen in the caecum and the ascending colon never encircled the lumen completely and had no fixed location; in contrast, a prominent fold, with a semilunar or circular shape, was constantly found at the ICJ distal border. Considering its location and morphology, this fold should correspond to the 'ileocaecal fold' previously described by other endoscopists, ${ }^{5-7}$ and to the 'Busi's sphincter' seen by radiologists. ${ }^{2}$

A prominent fold with a similar shape was also constantly found at the CCJ level in stimulated surgical specimens. This finding contrasts with published data based on necropsy material, reporting on the morphology of the ICJ and the neighbouring areas. ${ }^{8-11}$ In fact, these data only refer to thin and short structures (frenula) anchoring the ICJ lips to the adjacent caecocolonic wall. This contrast, however, might be apparent, as our material had been stimulated immediately after surgery by using a procedure that permitted the maintenance of intestinal morphology thus mimicking a physiological condition. In contrast, frenula might be the postmortem equivalent of both 'ileocaecal fold' (endoscopists' finding) and 'Busi's sphincter' (radiologists' finding). To avoid confusion in nomenclature and considering the anatomical location of this fold 


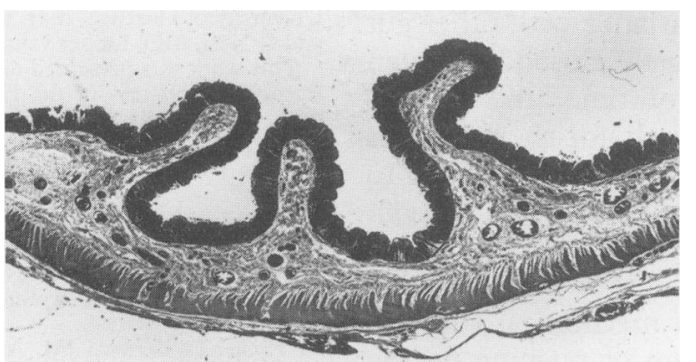

Figure 5: Longitudinal section of the CCF wall proximally to the CCF. Haematoxylin and eosin, original magnification $\times 7$.

endoscopically and macroscopically detected, we suggest that it should be called 'caecocolonic fold' (CCF).

Notwithstanding its macroscopic shape (semilunar or circular), the CCF showed histological features similar to those of the 'crescent-like folds' described in the ascending, transverse, and sigmoid colon, both in surgical and necropsy material, ${ }^{16}$ but completely different from those of the caecocolonic sphincter of other mammals, such as the dog. ${ }^{1}$ In fact, the CCF contained all the colonic layers (mucosa, submucosa, circular and longitudinal muscle layers, and serosa), but differently arranged in comparison with those of the 'crescent-like folds' and the canine caecocolonic sphincter. In fact, we found: (a) the circular muscle layer of the fold with a constant thickness, similar to that of the neighbouring areas, but made up of two differently arranged portions, (b) the longitudinal muscle layer showing a triangular shape, instead of forming a loop or a bridge. Moreover, we could show that, at variance with the crescent-like folds, either cutting the serosa or the taenia, the CCF did not disappear and maintained its specific arrangement. Furthermore, the peculiar architecture of the circular muscle layer of the stimulated CCJ

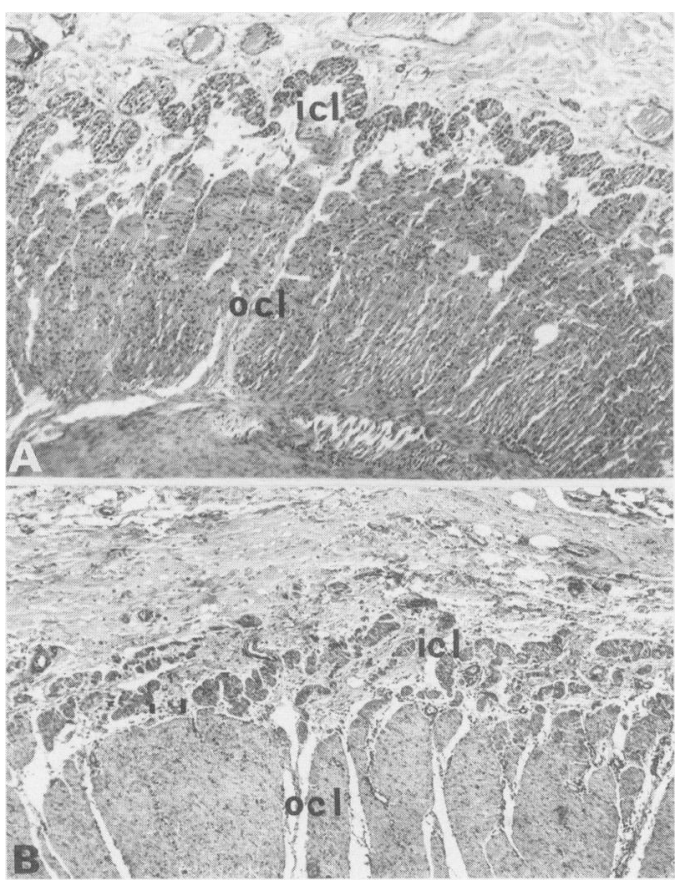

Figure 6: Detail of the circular muscle layer of the CCF icl: Inner circular layer, ocl: outer circular layer. $(A)$

Haematoxylin and eosin, (B) PAS, original magnification $\times 50$. was found identical to that previously described in the unstimulated $\mathrm{CCJ}^{13}$ and to that of the ICJ (present results).

Endoscopic findings also allowed us to see that the CCF can contract independently of colonic peristalsis. In fact, a spontaneous and an evoked spasm could be seen in $5 \%$ and in $47 \%$ of the examined patients, respectively. All these patients had a circular shaped CCF and the spasm was so intense to close the CCJ lumen completely and permitted the ICJ to move towards the caecum. Peristaltic waves coming from the caecum neither preceded nor followed this spasm along the ascending colon. These findings show that the CCF spasm is (a) independent of the colonic peristalsis, (b) circumscribed to the CCJ region, and (c) of a sphincteric type. To have a more precise evaluation of the CCJ motile activities, manometric studies should be performed.

Unfortunately, the manometric device does not give adequate information, as it does not maintain a fixed position in the caecum for a long period of time. Our findings, however, seem to be in agreement with the radiological finding of a circumferential contraction, at the CCJ level, the so called Busi's sphincter, after barium meal or enema, or both.

Macroscopically, a CCF completely occluding the CCJ lumen could be seen stimulating its appearance and contraction by filling the caecum through the terminal ileum in surgical specimens representative of the entire ileocaecocolonic region. Thus, both endoscopic and anatomical findings confirmed the occurrence of a circumferential and sphincteric contraction at the CCJ.

The presence at the CCJ of a circular muscle layer with a smooth muscle cell population ${ }^{13}$ and innervational patterns ${ }^{14} 15$ different from those of neighbouring areas, might be at the basis of the CCF motile behaviour. CCJ muscular and innervational patterns are similar to those described for ICJ in humans and large mammals ${ }^{1141718}$ (see present results). Presumably, as well as at the ICJ, the inner portion of the circular muscle layer of the CCF is responsible for the closure of the CCJ lumen. Therefore, similar to that proposed for the ICJ on the basis of morphological and functional findings ${ }^{11} 17-21$ these two junctional areas might play a sphincteric part.

In conclusion, our data suggest that the $\mathrm{CCJ}$ might be a region in which a sphincteric

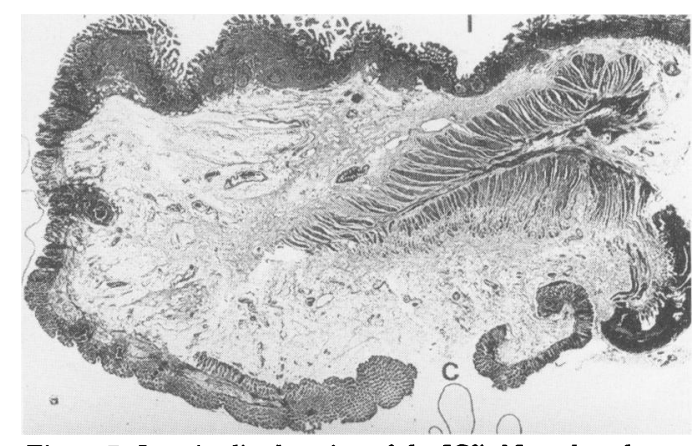

Figure 7: Longitudinal section of the ICF. Note that the inner circular muscle layer is more developed on the colonic side. $i$ : Ileal mucosa, c: colonic mucosa. Haematoxylin and eosin, original magnification $\times 5$. 
activity occurs; but, at variance with other gut sphincters, which are mostly closed and open only for short periods of time, the CCJ is usually open and closes only for short periods of time (from two minutes and 40 seconds to five minutes and 22 seconds in our findings). Until now, the mechanisms underlying the CCJ closure remain unknown. According to our findings, however, a mechanical stimulation, by filling the caecum or pushing on the CCF wall, seems to increase the CCF prominence, up to the closure of the CCJ lumen, presumably because of the contraction of the inner portion of the circular muscle layer. The loss of any type of contractile stimula at death might explain the presence of structures like frenula rather than prominent folds in necropsy material.

Until now, the physiological relevance in the colonic motile behaviour of a sporadic sphincteric activity at the CCJ level is unknown, but we think that it should not be underestimated. In fact, such an activity may play an important part in the regulation of the motile activities of the ileocaecocolonic region (that is, genesis of colonic mass movements, ileal flow accommodation, caecum filling and emptying).

Hypothetically, a CCJ malfunction might accompany or characterise some clinical manifestations, or both. Recent data, reporting a remarkable involvement of the CCJ in two different functional disorders of the colonic motility, might be in favour of this hypothesis. In fact, a circular shaped CCF was present in a high percentage of patients with irritable bowel syndrome, who underwent the same endoscopic procedure used in this study. In these patients the CCF showed spasms either spontaneous or evoked more prolonged than in control cases (Manneschi L, personal observation). Furthermore, the CCJ of patients affected by chronic idiopathic constipation had a myenteric plexus with a reduced number of neurons and a low density and percentage of both VIPergic and nitrergic neurons. ${ }^{22}$

This work was supported by a grant from the Italian Ministry of University and Scientific and Technologic Research. The authors thank Professor C Cortesini, Chief of Surgery Clinic,
University of Florence, Italy, for supplying fresh resected bowel and $G$ Nesi, $M D$, for her valuable language assistance.

This work was presented as a preliminary report at the 2nd Thepen Congress of the European Association of Clinical Anaper 1993; 15: 236).

1 Pelckmans PA. Study on the sphincteric nature of the ileocecal junction: a morphological and pharmacological approach (thesis). Antwerp: University of Antwerp, 1988.

2 Grilli A. Compendio di radiologia medica. Rome: SEU, 1968: 821-30.

3 Hickel L, Bernard JM. Anatomo-physiologie radiologique du gros intestin normal. Semaimes Hôpitalières 1963; 39; du gros

4 Torsoli A, Ramorino ML, Crucioli V. The relationships between anatomy and motor activity of the colon. $\mathrm{Am} \mathcal{F}$ Dig Dis 1968; 13: 462-7.

5 Cotton PB, Williams CB. Practical gastrointestinal endoscopy. Oxford: Blackwell, 1990: 209-11.

6 Schiller KFR, Cockel R, Hunt RH. A colour atlas of gastrointestinal endoscopy. London: Chapman, 1986: 181-2.

7 Sivak MV. Gastroenterologic endoscopy. Philadelphia: Saunders, 1987: 861-5.

$8 \mathrm{Di}$ Dio LJA, Anderson MC. The 'sphincters' of the digestive system. Baltimore: Williams and Wilkins, 1968.

9 Rutherford AH. The ileo cecal valve. London: HK Lewis, 1914.

10 Fit I. The structure and the development of the ileocolic valve and its frenula. Indian $\mathcal{f}$ Med Res 1956; 44: 361-73.

11 Quiglei FM, Phillips SF. The ileocecal (ileocolonic) sphincter. $Z$ Gastroenterol 1983; 21: 47-55.

12 Di Dio LJA, Geocze S, Ferrari Jr AP. Endoscopic study of the ileal papilla in a normal case and in patients under pathological conditions. Ann Anat 1994; 176: 263-8.

13 Faussone-Pellegrini MS, Pantalone D, Cortesini C. Morphological evidence for a cecocolonic junction in man and functional implications. Acta Anat 1993; 146: 22-30.

14 Faussone-Pellegrini MS, Bacci S, Pantalone D, Cortesini C. Distribution of VIP-immunoreactive nerve cells and fibers in the human ileocecal region. Neurosci Lett 1993; 157: 135-9.

15 Faussone-Pellegrini MS, Bacci S, Pantalone D, Cortesini C, Mayer B. Nitric oxide synthase immunoreactivity in the human ileocecal region. Neurosci Lett 1994; 170 261-5.

16 Torsoli A, Crucioli V, Young AC. On the structure of colonic folds. Rendiconto Riuniomi Radiologico-Gastroenterologiche 1969; 1: 195-202.

17 Gazet JC, Giarrett RJ. The ileocaecocolic sphincter. $\mathrm{Br} \mathcal{F}$ Surg 1964; 51: 368-70.

18 Phillips SF. Sphincters of the gastrointestinal tract: functional properties. In: Daniel EE, Tomita T, Tsuchida $S$, Watanabe $M$, eds. Sphincters normal function-changes in diseases. Bocha Baton: Press Inc, 1992: 8-27.

19 Ulin AW, Shoemaker WC, Deutsch J. The ileocecal valve and papilla. Observations relating to pathophysiology of acute colon obstruction. Arch Intern Med 1956; 97: 409-20.

20 Scheye T, Dechelette P, Vanneuville Tanguy A, Chazal J, Amrane M. Etude anatomique et histologique de la valve caecale humaine. Aspects evolutivs en fonction de l'âge. Bull Assoc Anat (Nancy) 1983; 67: 485-99.

21 Bogers JJ, Vanmark E. The ileocaecal junction. Histol Histopathol 1993; 8: 561-6.

22 Faussone-Pellegrini MS, Matini P, Cortesini C, Mayer B, Infantino A, Lise M. Chronic idopathic constipation and distribution of VIP and nitric oxide synthase (NOs) immunoreactive neurons at the various colonic levels. immunoreactive neurons at the various colonic 